\title{
LAS REFORMAS AL IMPUESTO AL VALOR AGREGADO Y SU EFECTO EN LA ECONOMÍA DEL CONSUMIDOR EN LA PROVINCIA DE SANTA ELENA. AÑ̃ 2016.
}

Nelson Cristóbal, Margarita Panchana, Mónica Tomalá, Luis Asencio. 


\title{
Las reformas al impuesto al valor agregado y su efecto en la economía del consumidor en la provincia de Santa Elena. Año 2016
}

\section{Reforms to the tax to the added value and its effect on the economy of the consumer in the province of Santa Elena. Year 2016}

\author{
Nelson Asencio Cristóbal ${ }^{1}$, Margarita Panchana ${ }^{1}$, Mónica Tomalá $\mathrm{Ch}^{1}$. Luis Asencio Cristóbal ${ }^{2}$ \\ Facultad de Ciencias Administrativas \\ Universidad Estatal Península de Santa Elena \\ Universidad de Guayaquil ${ }^{2}$ \\ nasencio@upse.edu.ec
}

\begin{abstract}
Resumen
El objetivo del presente reporte es elaborar un análisis de las reformas tributarias del IVA implementadas en el país en el año 2016 y referir su impacto en la economía del consumidor en la provincia de Santa Elena. Desde una perspectiva teórica, se define la estructura impositiva del país y su importancia en la generación de ingresos públicos para la consecución objetivos sociales; así como también los fines que conllevan las reformas tributarias tendientes a regular la economía, fomentar el empleo, incentivar la producción, la demanda y el consumo. En la investigación que dio origen a este artículo se aplicaron procedimientos metodológicos deductivo, cuantitativo, y documental provenientes de fuentes oficiales y técnicas de investigación que permitieron obtener información relevante para el análisis descriptivo de las reformas, cuyos resultados reflejan la necesidad de que el gobierno, previa a la adopción de estas medidas, realice con profundidad un estudio socio-económico, que permita valorar las incidencias que tendrían en el sector productivo del país y en el consumido específicamente. En la investigación se describen las últimas reformas tributarias del IVA para luego referir su efecto general en el consumo de bienes y servicios del consumidor peninsular.
\end{abstract}

Palabras clave: tributos, reforma tributaria, el iva, carga tributaria, consumidor, cultura tributaria

\begin{abstract}
The objective of the present report is to develop an analysis of VAT tax reforms implemented in the country in the year 2016 and see its impact on the economy of the consumer in the province of Santa Elena. From a theoretical perspective, defines the country's tax structure and its importance in the generation of revenue to achieve social objectives; as well as also the purposes involving tax reforms aimed at regulating the economy, promoting employment, encourage the production, demand and consumption. In the research that gave origin to this article is applied procedures methodological deductive, quantitative, and documentary from of sources official and technical of research that allowed obtain information relevant to the analysis descriptive of them reforms, whose results reflect the need of the Government, prior to the adoption of these measures perform with depth a study partner-economic, that allow rating them incidents that would have in the sector productive of the country and in the consumed specifically. In the research is described the latest reforms tax of the VAT to then refer its effect general in the consumption of goods and services of the consumer peninsular
\end{abstract}

Keywords: taxes, tax reform, vat, tax burden, consumer, tax culture 


\section{Introducción}

En todo Estado, los impuestos son fuente económica importante para el cumplimiento de sus principales objetivos sociales, por lo que requiere de una estructura y normas legales que le permitan dictaminar políticas fiscales necesarias para el logro de sus fines, entre las que se destacan las políticas tributarias, las mismas que conllevan reformas que cambian sustancialmente la estructura tributaria, buscando a través de ella aumentar o disminuir la cantidad de dinero que recibe el Estado por concepto de impuestos, a fin de solventar el gasto público en beneficio de la sociedad.

Los gobiernos adoptan cambios en el sistema impositivo a causa de factores endógenos y exógenos, por tanto las reformas tributarias constituyen un importante instrumento de política fiscal, que a más de brindarle recursos públicos al Estado permite redistribuir el ingreso, fomentar la producción y empleo; regularizar la carga tributaria, disminuir la evasión tributaria entre otros fines.

De acuerdo a la CEPAL (2016) "el año 2015 estuvo marcado por la pérdida de ingresos provenientes de recursos naturales no renovables, ese declive fue contrarrestado en parte en varios países por aumentos de los ingresos tributarios. En promedio, América Latina logró aumentar su presión tributaria en 0,2 puntos porcentuales del PIB. Se destacan en particular Chile (0,5 puntos porcentuales del PIB), México (2,2 puntos porcentuales) y el Ecuador (1,0 puntos porcentuales), donde las reformas y las medidas recientes generaron mayores ingresos..., entre los que más aumentaron sus ingresos tributarios durante ese año se destacan Chile (0,5 puntos del PIB), México (2,2 puntos) y el Ecuador (1,0 puntos), donde las reformas y medidas recientes generaron mayores ingresos.

Sim embargo el desempeño del impuesto sobre el valor agregado ha sido menos dinámico entre los países de la región en 2015. En particular, se registran descensos en el Brasil $(-6,7 \%)$, el Ecuador $(-2,8 \%)$, Guatemala ($1,3 \%)$ y el Perú $(-0,9 \%)$ como consecuencia de una desaceleración económica, incluso del consumo privado en algunos casos.

En el caso de los cambios en el impuesto sobre el valor agregado, las medidas aprobadas también se concentran en modificaciones de las bases imponibles. En la mayoría de los países (menos en Colombia y Guatemala) aumentaron los ingresos provenientes de la tributación indirecta, debido principalmente a un mejor desempeño de los impuestos generales sobre bienes y servicios, y en particular, del impuesto sobre el valor agregado. En el caso del IVA, en esos países tuvo lugar una serie de modificaciones tributarias, como: i) el aumento de la tasa general en Honduras (del 12\% al
15\%), México (del 15\% al 16\%), Panamá (del 5\% al $7 \%$ ) y la República Dominicana (del $16 \%$ al $18 \%$ ); ii) el establecimiento de tasas mayores de IVA para determinados bienes y servicios (Honduras y Panamá), y iii) una ampliación de la base imponible en el Ecuador (se comienza a tributar sobre los servicios financieros)" (1)

En el Gobierno de Ecuador ha realizado varias reformas tributarias en los últimos años, tanto netamente recaudatorias, así como para preservar el medio ambiente y actualmente aquellas para la reactivación económica de las zonas afectadas por el fenómeno telúrico del mes de abril pasado, aumentando el IVA del 12 al 14\% temporalmente, que con la caída del precio del petróleo, las desventajas en las relaciones de intercambio comercial y la devaluación del dólar de países vecinos, han empeorado la realidad económica del país.

Esta investigación tiene como propósito analizar las últimas reformas tributarias del IVA y su efecto en la economía del consumidor peninsular frente a la subida de precios de los bienes y servicios, que ligados a los problemas del desempleo declina la situación económica de los ciudadanos.

El presidente del Colegio de Economistas del Guayas, considera que el alza de impuestos va a generar una disminución del consumo de los ciudadanos (más cuando ha caído el índice de confianza del consumidor), lo que implicará menos ventas, menos producción y menos recaudación. (2)

Así también, el vocero del Observatorio de la Política Fiscal, consideró que, si bien el alza de dos puntos en el IVA puede considerarse "moderada", el problema es que se generan efectos en cascada en toda la economía. Además consideró que para los sectores más pobres, un alza de centavos puede afectar el consumo. Explicó que aunque hay productos, como alimentos y combustibles, que no se gravarán con IVA directamente, sí pueden tener un incremento porque en la cadena de comercialización suben los precios de los repuestos de los transportes, aceites y otros. (3)

En este sentido, se puede colegir que el IVA es uno de los instrumentos de mayor importancia con el que cuenta el Estado para obtener ingresos y financiar el desarrollo económico, la equidad y la redistribución del ingreso, por lo que resulta importante examinar el grado de impacto que tienen las reformas a los impuestos en la economía del consumidor. En la provincia de Santa Elena, cuya actividad productiva se basa principalmente en el comercio, la pesca y artesanía, en el año 2014 se recaudó por concepto de impuestos \$ 24.9 millones en el 2015 \$ 24.7 millones y en el año 2016 \$ 24.6 millones, de este monto por IVA 
se recaudó \$ 10,2 millones y 14,9 millones y 10,1 millones en los años indicados (4)

\section{Objetivo general.}

Determinar el grado de influencia de las reformas del Impuesto al Valor Agregado, en la economía del consumidor de la Provincia de Santa Elena.

\section{Objetivos Específicos.}

- Determinar las causas y efectos de las reformas tributarias del IVA

- Describir la importancia de las reformas tributarias y evaluar su incidencia en la economía del consumidor peninsular

- Establecer el grado de cultura tributaria de la ciudadanía peninsular

\section{Materiales y métodos}

Al considerar la necesidad de diagnosticar la incidencia de las reformas tributarias del IVA en la economía de la ciudadanía peninsular, se aplicó una metodología de tipo cuantitativo, descriptivo y analítico, con referencia bibliográfica y secundaria de tipo estadístico provenientes de fuentes oficiales y técnicas de investigación cuyos datos tabulados permitieron obtener información relevante para realizar un análisis descriptivo y comparativo de los resultados en concordancia con los objetivos planteados.

La población con la que se trabajó en el presente estudio es de 130270 habitantes que corresponden a la Población Económicamente activa actual de la provincia de Santa Elena de acuerdo a los datos proyectados por el INEC y aplicando una muestra de tipo probabilístico, se realizó encuestas a 398 personas, $55 \%$ corresponden al género masculina y la diferencia al género femenino, seleccionadas aleatoriamente y que acuden a los mercados de bienes y servicios a realizar sus adquisiciones, técnica que permitió validar la hipótesis planteada en el desarrollo de estudio, basándose en un cuestionario con preguntas estandarizadas para su fácil tabulación. Se utilizó el sistema de distribución de frecuencias y la representación gráfica para analizar e interpretar los resultados.

\section{Desarrollo}

Contribuir con el Estado a través de los tributos es un compromiso y obligación de los ciudadanos, recursos que servirán para financiar las necesidades sociales .La tributación es un concepto que se articula alrededor de algunos principios básicos, que proviene de varios enfoques: económico, jurídico, administrativo, social, entre otros, por lo que antes de inducir en el propósito de esta investigación es necesario remitirnos a varias concepciones en materia impositiva.
Para Villegas (2001) tributo es" una prestación de dinero que el Estado exige en el ejercicio de su poder de imperio sobre la base de la capacidad contributiva en virtud de una ley, y para cubrir los gastos que le demande el cumplimiento de sus fines" (p.67)

Según Fleiner, citado por el jurista paraguayo Carlos A. Mersan, en su obra Derecho Tributario define al tributo como: "prestaciones pecuniarias que el Estado u otros organismos de Derecho público exigen en forma unilateral a los ciudadanos para cubrir las necesidades económicas". (5). De acuerdo a la legislación ecuatoriana se entiende por tributos: "los impuestos, tasas y las contribuciones especiales y de mejoras y tienen como finalidad recaudar ingresos públicos, estimular la inversión, la reinversión, el ahorro y su destino hacia los fines productivos y de desarrollo nacional; atenderán a las exigencias de estabilidad y progresos sociales y procurarán una mejor distribución de la renta nacional" (6)

En base a estas concepciones podemos definir de forma general que los tributos son aquellas prestaciones de carácter económico exigidas por el Estado a través de la administración tributaria a los sujetos pasivos o contribuyentes en potestad de una ley con la finalidad de financiar el gasto público y satisfacer los requerimientos del desarrollo nacional. Los tributos se pueden gravar sobre el consumo o el gasto de los contribuyentes, sobre sus fuentes de ingreso económico y por concepto de un servicio prestado por el Estado.

\section{Impuesto al Valor Agregado}

Los impuestos son tributos que cobra el estado a los contribuyentes en forma general y obligatoria y que estos deben pagarlos en virtud de poseer un patrimonio, ingresos o realizar actividades de compra venta de bienes y servicios. Ente los impuestos más importantes en la generación de ingresos públicos se encuentran: El impuesto a la Renta; el IVA, el impuesto a los consumos especiales, el impuesto a la salida de divisas, los impuestos ambientales, entre otros.

Maldonado (2005, p.1) define al Impuesto al valor agregado (IVA) como un tributo indirecto que grava a las ventas y demás transferencias de bienes y a la prestación de algunos servicios. Su nombre se atribuye porque grava a todas las tapas de comercialización, dando lugar al hecho generador en cada una de ellas.

Para Paz y Miño(2015) el impuesto al valor agregado grava "sobre el valor de la transferencia de dominio(compra/venta) o a la importación de bienes muebles; así como a los de autor, propiedad industrial y derechos conexos; y el valor de los servicios prestados"(p.45) 
De acuerdo a la norma tributaria del país, el Impuesto al Valor Agregado (IVA) grava al valor de la transferencia de dominio o a la importación de bienes muebles de naturaleza corporal, en todas sus etapas de comercialización, así como a los derechos de autor, de propiedad industrial y derechos conexos; y al valor de los servicios prestados.

De acuerdo a lo expresado podemos decir en términos generales el IVA es un impuesto indirecto que grava a las transacciones de bienes y servicios, es decir grava sobre el consumo de bienes y servicios sin considerar la capacidad económica directa de los contribuyentes. Existen dos tarifas básicas para este impuesto que son $0 \%$ y tarifa $12 \%$.

El IVA como parte de los ingresos tributarios, es el impuesto más gravitante y determinante en los ingresos fiscales de la mayoría de los países de América Latina. En los últimos años el presupuesto general del Estado ecuatoriano se financia en un mayor porcentaje con la recaudación de impuestos directos e indirectos, en donde el IVA es el tributo con más recaudación, seguido del impuesto a la renta, el ICE entre otros. Según datos del SRI el IVA en el 2014 represento el $48 \%$, en el 2015 el $50 \%$ y para el 2016 el $48 \%$ del total de la recaudación efectiva.

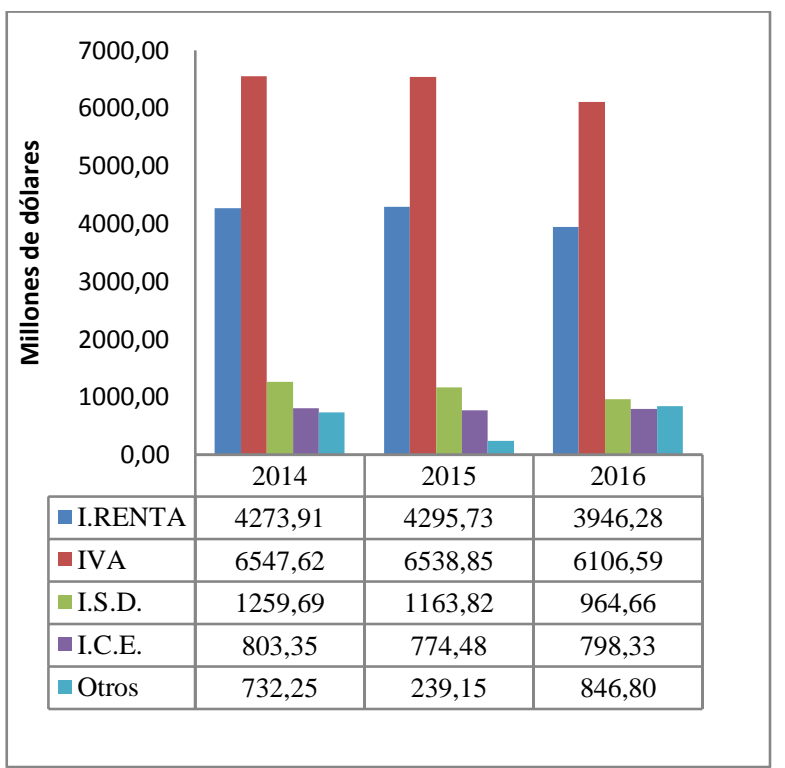

Figura 1. Recaudación efectiva de los principales impuestos

\section{Reforma tributaria}

Reforma tributaria es una "modificación de la estructura de impuestos, la cual determina quién o quiénes deben o no pagar estos, así como el motivo de ello, la cantidad que deben pagar dependiendo de las políticas de los gobiernos" (Camposano, 2012, p.16)

Una reforma tributaria "cambia uno o varios aspectos de la estructura tributaria (impuestos a las personas y/o empresas) buscándose, a través de ella, aumentar o disminuir la cantidad de dinero que recibe el Estado por concepto de impuestos" (7)

De las acepciones anteriores podemos manifestar que, las reformas tributarias implican un cambio estructural en el sistema tributario de un país, y por tanto los sectores productivos y la economía del consumidor se ve afectada por estas medidas. El actual régimen ha implementado 15 reformas tributarias, la más significativa durante este año fueron las reformas presentadas a partir del terremoto, ocurrido el pasado 16 de abril del presente año, 'la misma que dispuso contribuciones solidarias y un alza de 2 puntos al Impuesto al Valor Agregado, pasando de una tarifa de $12 \%$ a $14 \%$, y que produce un mayor costo en los bienes y servicios gravados con este impuesto. .La reforma al IVA entro en vigencia el 1 de junio del 2016, con un periodo de duración de un año, medida que no regirá en las provincias de Manabí y Esmeraldas por ser zonas afectadas.

\section{El Comportamiento del consumidor}

Consumidor.- Es el individuo que adquiere bienes y servicios producidos por las empresas por medio del mercado y que está dispuesto a pagar el valor asignado con el fin de satisfacer sus necesidades. Las ciencias sociales han desarrollados diversas teorías que tratan de explicar el comportamiento del individuo frente a sus necesidades. "Al respecto, Arellano y otros (2009), señalan que el concepto de comportamiento refiere la dinámica interna y externa del individuo, o grupos de ellos, originada cuando buscan satisfacer sus necesidades mediante bienes y servicios. Al efecto, definen el comportamiento del consumidor como el proceso de decisión y la actividad física realizada al buscar, evaluar, adquirir, usar o consumir bienes, servicios e ideas para satisfacer sus necesidades"(.Tirrito,2015,p.1).

Sin embargo, es importante manifestar que el enfoque económico del comportamiento humano ha estado orientado a la racionalización de los problemas mediante el empleo de fórmulas matemáticas para simular la conducta de los individuos, de lo que se ha derivado su denominación de teorías o modelos normativos. Este enfoque también considera que la información que posee el individuo es completa, perfecta y oportuna, situación que le permite optar por la mejor alternativa y maximizar su posición frente a los demás

El comportamiento del consumidor está ligado a una acción económica. (Lambin \& Peeters, 1983) citado por Timana y Pazo(2014) manifiestan que la economía, como ciencia social, estudia la forma en la cual los seres humanos utilizan los recursos escasos para obtener diversos bienes y servicios y la distribución que 
realizan para su consumo presente y futuro, se sustenta en que el individuo, en calidad de consumidor, elige entre las diferentes alternativas que le ofrece el mercado de manera racional y trata de alcanzar un cierto bienestar a partir de sus limitados recursos (p.22)

Respecto a la teoría de la conducta del consumidor, Pindyck y Rubinfeld (2009) refieren que es una "descripción de cómo asignan los consumidores su renta a los diferentes bienes y servicios para maximizar su bienestar. Además sostienen que para comprender la conducta del consumidor hay que considerar los siguientes pasos: las preferencias, las restricciones y las elecciones" (p.76 ) es decir que las decisiones de consumo de las personas están en función de sus gustos o preferencias, a su presupuesto y al precio de los bienes y servicios

\section{Cultura tributaria}

"Maksvytienė y Šinkūnienè (2012) definen la cultura tributaria como un fenómeno social que se expresa a través de las personas que participan en la creación del sistema tributario, la cual se percibe a través de su actitud en el sistema de impuestos. También precisan que es la totalidad de las relaciones fiscales que se forman por la cultura nacional para la optimización de ingresos fiscales $y$ que abarca actitud y comportamiento de los individuos en el sistema de impuestos, influenciados por el nivel de desarrollo de un país, la situación social y el sistema de educación" (Tamana y Pazo, 2014, p.47)

La cultura tributaria es el "conjunto de información y el grado de conocimiento que un país tiene sobre los impuestos, o el cúmulo de percepciones, criterios hábitos y actitudes que la sociedad tiene respecto a la tributación" (8)

\section{Las reformas tributarias del IVA en el año 2016}

Debido a la crisis económica que atravesaba el país, a causa de la caída del precio del petróleo y la apreciación del dólar principalmente, lo que nos resta el nivel de competitividad con otros países el Gobierno Nacional opto por crear en el mes de abril la Ley Orgánica para el equilibrio de finanzas públicas, a fin de optimizar y perfecciona el sistema tributario fortaleciendo la redistribución, y garantizar un sistema fiscal justo y equitativo, además de generar una recaudación extraordinaria de más de 300 millones de dólares en el 2016

Con esta ley se crean incentivos importantes para fomentar el uso de tarjetas de crédito, tarjetas de débito y moneda electrónica, instrumentos de suma importancia para mantener el actual régimen monetario del país. Además plantea la ventaja de que los contribuyentes que utilicen el dinero electrónico tengan la posibilidad de la devolución de dos puntos porcentuales del IVA y los que utilicen tarjetas de crédito o débito tengan la devolución de un punto porcentual de IVA.

Al aplicarse la ley, en la práctica, resulta que si se ha pagado con dinero electrónico una compra de 100 dólares, el IVA efectivo sería de 10 dólares, cuando se trata de dinero electrónico, y si la compra se la hace con tarjetas de débito o de crédito, sería de 11 dólares (9)

En el mes de mayo del 2016 con la creación de la Ley de Solidaridad, el gobierno aumenta en dos puntos porcentuales el Impuesto al Valor Agregado con lo cual pasa del 12 al 14\% desde junio por un año como consecuencia del desastre natural ocurrido el 16 de abril del presente año, siendo la cuarta modificación en los últimos 20años: del $5 \%$ al $6 \%$ en el año 1993, del $6 \%$ al $10 \%$ en 1986 , del $10 \%$ al $12 \%$ en el 2000 y en este año 2016 al 14\%. La Ley establece que al usar dinero electrónico habrá una devolución de cuatro puntos del IVA.

De acuerdo con los datos ilustrados en la figura $1 \mathrm{y}$ de la tabla 1 se puede colegir que la recaudación del IVA en el 2016 disminuyó un $6,61 \%$ respecto al año anterior y en relación a nuestra provincia también registro un descenso del 7,76\%. Destacando que a nivel nacional la disminución hubiese sido mayor si no consideramos los 402 millones de dólares recaudados por IVA para destinarlo a las provincias afectadas por el sismo, deduciendo que la crisis ocasionada por factores internos y externos afecto a la economía del país, a la producción y al aumento del desempleo, esto se explica porque subir los precios de los bienes e insumos por efectos de las reformas, desincentiva la producción y el consumo afectando la recaudación tributaria.

Tabla 1. Recaudación tributaria del IVA Provincia de Santa Elena (Dólares)

\begin{tabular}{|l|c|c|c|}
\hline \multicolumn{1}{|c|}{ Años } & 2014 & 2015 & 2016 \\
\hline La Libertad & 3.416 .205 & 3.680 .071 & 3.111 .681 \\
\hline Santa Elena & 3.953 .878 & 4.213 .661 & 4.365 .074 \\
\hline Salinas & 2.839 .450 & 3.087 .968 & 2.652 .805 \\
\hline Total & 10.209 .533 & 10.981 .700 & 10.129 .560 \\
\hline
\end{tabular}

Fuente: SRI

\section{Resultados}

Las reformas tributarias del IVA han generado reacciones contrarias desde la perspectiva de un impacto social, cuyos resultados del levantamiento de la información con las técnicas de investigación aplicadas permiten analizar el problema investigado, exponiendo a continuación los resultados más relevantes. 
¿El aumento del IVA afecta a las personas de escasos recursos económicos?

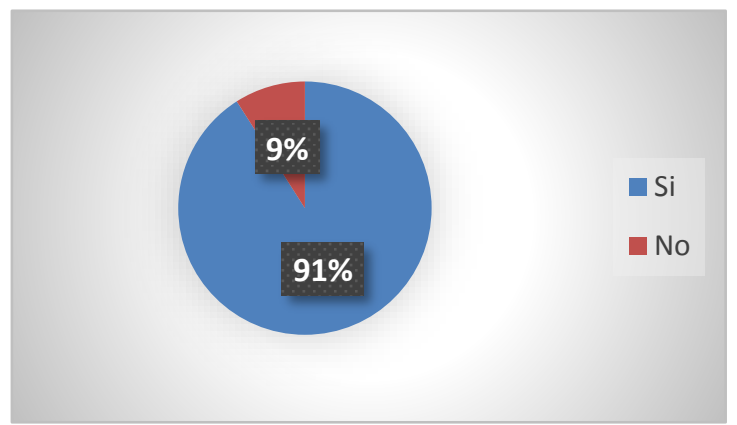

Figura 2. EI IVA afecta a los recursos económicos

Del total de consumidores encuestados, 362 personas equivalente al $91 \%$ respondieron que el aumento del IVA del $12 \%$ al $14 \%$ si afecta a las personas de escasos recursos económicos, mientras que 36 personas equivalente al $9 \%$ respondieron que las personas de escasos recursos no se verían afectadas a partir de una nueva reforma. Evidenciando la preocupación de la mayoría de los encuestados debido a que con el desempleo su situación económica se agudiza

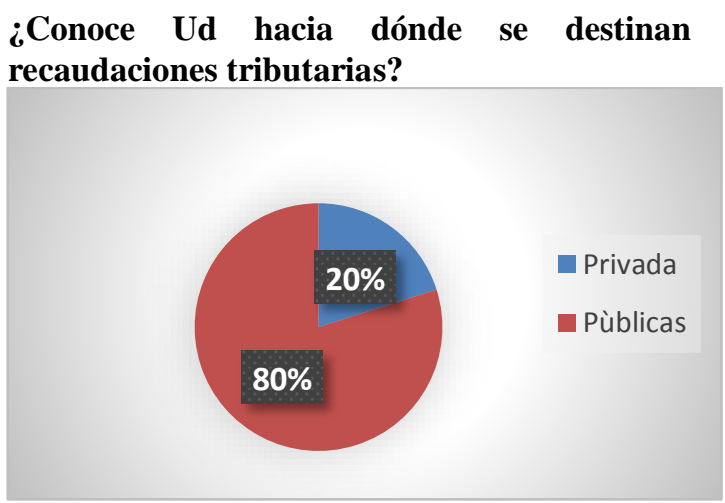

Figura 3. Destinos de las recaudaciones

Según los datos registrados, 318 personas, equivalente al $80 \%$ de los encuestados, respondieron que las Recaudaciones Tributarias de destinan al gasto social y obras públicas, mientras que el $20 \%$, es decir 80 personas respondieron que se destinan a las actividades privadas. Es decir que las personas conocen que el pago de sus impuestos están destinados para el gasto público: Salud, Educación, vivienda, carreteras, etc y que benefician a la economía del país, sin embargo otros opinan que son destinados para otros menesteres de índole particular.

¿Está usted de acuerdo con el incremento del IVA al $14 \%$ establecido en La Ley de Solidaridad?

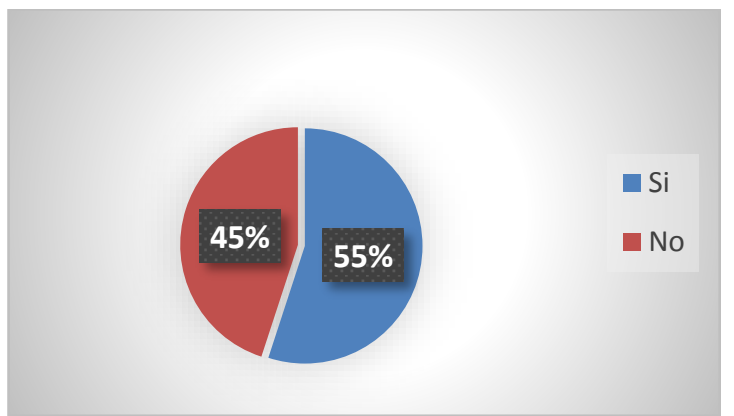

Figura 4. EI IVA y La Ley de Solidaridad

Las opiniones de los consumidores encuestados estuvieron muy divididas, el $45 \%$, es decir, 179 personas respondieron que No estaban de acuerdo con el incremento el IVA, mientras que un 55\%, equivalente a 219 personas encuestadas respondieron Sí apoyaban esta imposición tributaria considerando que tiene vigencia un año y que es para ayudar a las provincias en desastre, mientras otras manifiestan no estar de acuerdo porque más encaren el costo de los bienes y servicios.

Tabla 2. Reforma Tributaria ¿Según su criterio hacia donde se debería orientar una reforma tributaria?

\begin{tabular}{|l|c|c|}
\hline \multicolumn{1}{|c|}{ Valoración } & No .encuestados & Porcentaje \\
\hline Subir los impuestos & 36 & $9 \%$ \\
\hline Bajar los impuestos & 203 & $51 \%$ \\
\hline $\begin{array}{l}\text { Mantener los } \\
\text { impuestos }\end{array}$ & 147 & $37 \%$ \\
\hline $\begin{array}{l}\text { Eliminar los } \\
\text { impuestos }\end{array}$ & 12 & $3 \%$ \\
\hline TOTAL & 398 & $100 \%$ \\
\hline
\end{tabular}

De acuerdo a los datos recabados, $9 \%$ de personas encuestadas (36) opinaron que las reformar deberían orientarse a subir los impuestos, un 51\% (203) manifestó que estos deberían bajar, Así también un $37 \%$ (147) respondió que se deberían crear impuestos y finalmente un $4 \%$ (12) dijo que estos deberían eliminarse. Interpretando que frente a los fenómenos adversos el gobierno debería aplicar otras estrategias para financiar y generar los ingresos fiscales y atender el gasto público, debido a que el mayor peso de los impuestos finalmente recae sobre el consumidor.

¿ Cual es el grado de carga tributaria que pagan actualmente los consumidores? 


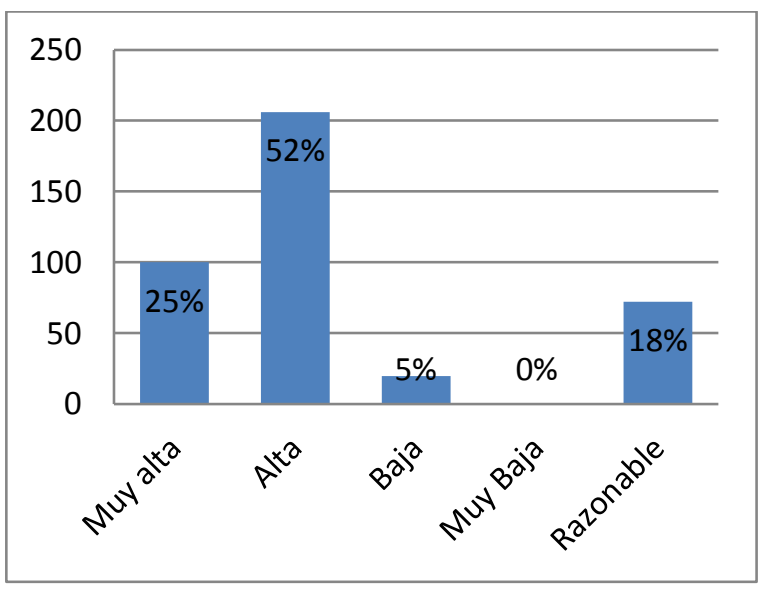

Figura 5. carga tributaria

El $52 \%$ de encuestados respondió que la carga tributaria es alta, un $25 \%$, por lo tanto, 35 personas mencionaron que es muy alta, otro $18 \%$ de las personas respondió que la carga tributaria es razonable, un $5 \%$ de los encuestados de la provincia de Santa Elena mencionaron que es baja y ninguna opción muy baja. Efectivamente estos datos demuestran que los consumidores tienen que hacer frente a las restricciones de sus ingresos y el precio de los bienes y servicios, debido a la subida de precios y con problemas de pleno empleo su ingreso no alcanza a cubrir sus necesidades básicas, por consiguiente con más pago de impuestos menos disponibilidad y capacidad de compra.

\section{Discusión}

La provincia de Santa Elena, es una de las tantas provincias que percibe el impacto del incremento del impuesto al valor agregado, ya que resulta inevitable dejar de consumir, sin embargo hay que acatar las leyes que a la vez, están encausadas a satisfacer necesidades sociales, y su recaudación adicional de dos puntos porcentuales serán para obras de infraestructuras de zonas afectadas por el desastre natural; aunque esta ley esté vigente solo por un año ha causado una gran disminución de la actividad productiva ,comercio y turismo

Existen criterios divididos por parte los consumidores peninsulares, mientras unos están conscientes que las reformas tienen como propósito el interés público otros piensan que las mismas deberían estar orientadas a bajar los impuestos, ya que una subida aumenta el precio de los bienes y servicios y con sus ingresos bajos no logran cubrir sus necesidades elementales, agudizando más el problema con aquellos que no tienen empleo. Al respecto de acuerdo a la ley un gran porcentaje de los bienes que conforman la canasta básica de acuerdo no gravan IVA

Sin embargo, el Estado debería implementar políticas públicas orientadas a fomentar e impulsar al sector productivo y a la inversión por ejemplo, exonerando impuestos a bienes y materias primas importadas, bajar las tasas de interés, etc. a fin de lograr una mayor productividad, a la vez permita fomentar el empleo, y una distribución equitativa de los ingresos públicos. Por otro lado, la tasa de desempleo nacional registro un aumento causado principalmente por el desastre natural, provocando un desequilibrio en la economía de estas zonas afectadas, incluida la península; para fines del 2016 la tasa registró el 5,8\% mayor al 4,76\% del año anterior.

\section{Conclusiones}

Las reformas tributarias constituyen un instrumento de política fiscal que procuran generar ingresos por concepto de recaudación impuestos, permitiendo al Estado atender el gasto público; también tienen con finalidad redistribuir los ingresos fiscales, fomentar la producción y generar fuentes de empleo. Sin embargo, el ciudadano peninsular exterioriza que las reformas afectan su economía encareciendo los bienes y servicios, situación que más se agudiza por la falta de empleo.

En el periodo de estudio se observa una disminución $2,68 \%$ en la recaudación efectiva de los impuestos, excepto el ICE y otros impuestos menores que registraron un ligero crecimiento, dicha disminución hubiese sido mayor si no se considera los recursos generados en aplicación a la Ley de Solidaridad. En la provincia de Santa Elena en el 2016 la recaudación del IVA disminuyó el 7,76\% respecto al 2015.

Los ciudadanos peninsulares poseen un grado de cultura tributaria aceptable toda vez que están conscientes que con el cumplimiento de sus obligaciones tributarias contribuyen a la economía del país, no obstante muchos manifiestan que éstas deberían orientarse a disminuir los impuestos a fin de no agudizar su economía familiar. En este sentido, el Estado debería optar por estrategias o políticas públicas que permitan lograr una mayor productividad de bienes y servicios, generar mayores ingresos públicos y una distribución equitativa del ingreso nacional.

\section{Referencias bibliográficas}

(1)CEPAL (2016) Panorama fiscal de América Latina y el Caribe. Naciones Unidas. Chile.

(2)Yumibanda, L. (2016)A comercio de Ecuador le preocupa alza en principales productos de venta. Recuperado de http://www.eluniverso.com/noticias/2016/04/03/nota/ 5499846/comercio-le-

(3)Carrera, J.(2016) Aumento del IVA del 12\% al 14\% regirá desde junio próximo en Ecuador Recuperado de http://www.eluniverso.com/noticias/2016/05/16/nota/ 5582889/aumento-iva-12-14- 
(4) www.sri.gob.ec/ Estadísticas de recaudación (5) Aguirre, R.(2009) Tributos en el Ecuador (2009) Recuperado;

http://www.derechoecuador.com/articulos/detalle/arch ive/doctrinas/derechotributario/2009/10/22/el-tributoen-el-ecuador

(6)Troya Jaramillo, J. V.(1984) "Estudios de Derecho Tributario”. Serie Estudios Jurídicos, Volumen 1, Quito: Edit. Corporación de estudios y publicaciones

(7)Villegas, H. (2001) Curso de derecho financiero y tributario. Argentina Ed.JJCPM Recuperado de http://www.academia.edu/8454470/Hector_Villegas_-

(8) Ortiz Efrain, J. (2009). Asesoría Fiscal. Ecuador. IVA Recuperado de http://www.mailxmail.com/cursofacturacion-cobros-pagos-impuestos/impuesto-valoragregado

(9) Bustos J.(2016)Reforma tributaria impulsa uso de dinero electrónico. Recuperado de http://www.eluniverso.com/noticias/2016/03/31/nota/ 5495943/reforma-tributaria-impulsa-uso-dineroelectronico

\section{Bibliografía}

1.-Balseca, M.(2012).Equidad y DesarrolloQuito SRI.

2.- Bocanegra, G. y Vásquez, M. (2009). La Conducta del Consumidor en una Economía Local. 56(11),1-20. 3.- Fergusson Leopoldo \& Suarez G." Política Fiscal: Un enfoque de tributación óptima ".Edit.Uniandes2011

4.- Maldonado P, H.(2005) Impuesto al Valor Agregado

5.- Mersan F.\& Carlos A., Derecho Tributario, 8a. edición, Editora Litocolor, Asunción, 1997,Pag.26 Recuperado.

file:///C:/Users/usuario/Downloads/30250-64587-1-

PB.pdf

6.- Ortiz Efrain, J. (2009). Asesoría Fiscal. IVA. Ecuador
7.- Paz y Miño, J., y Cepeda, M (2015). Historia de los Impuestos en el Ecuador. Quito: SRI-PUSE-THE.Pag.45 8.-Pindyck y Rubinfeld( 2009)Microeconomia,España Pearson Prentice Hall.

9.- Ruiz, Bocanegra Gastelum y Vázquez M (2009). La conducta del consumidor. eumed.net.

10.- Sistema de Rentas Internas del Ecuador, publicaciones varias sobre análisis de las reformas e informes de recaudaciones tributarias.2014-2016

11.-Servicio de Rentas Internas . (2012). En M. R. Moscoso, Equidad y desarrollo. Quito

12.- Timaná J. \& Pazo J (2014) Pagar o no pagar es el dilema: las actitudes de los profesionales hacia el pago de impuestos en Lima Metropolitana, Universidad ESAN, $2014 \quad$ Recuperado http://www.esan.edu.pe/publicaciones/Pagar\%200\%2 0no\%20pagar\%20para\%20Cecosami.pdf

13.- Troya José V: Estudios de Derecho Internacional Tributario, Pudeleco, Editores del Ecuador S.S, Marzo 2008.Ecuador

\section{Normas legales}

1.-Constitución de la República del Ecuador - R.O. \#. 449, 20 de octubre de 2008

2.-Ley Orgánica para el Equilibrio de las finanzas públicas R.O.\# 744 del 29 de abril del 2016

3.- Ley Orgánica de Solidaridad y corresponsabilidad ciudadana para la Reactivación y reconstrucción y Reactivación por el terremoto del 16 de abril del 2016.R.O \#759 - 20 de Mayo 2016

4.- Ley Orgánica de Régimen Tributario Interno Codificada. R.O.\#. 463 - 17.11.2004 